Pure and Applied Mathematics Quarterly

Volume 8, Number 4

$921-927,2012$

\title{
Support Theorems for Horocycles on Hyperbolic Spaces
}

\author{
Sigurdur Helgason \\ In Memory of S.S. Chern
}

\begin{abstract}
The horocycle transform on a hyperbolic space $H$ associates to each function on $H$ the integral over each horocycle in $H$. Here we prove a special support theorem for this transform.
\end{abstract}

\section{INTRODUCTION}

The Radon transform associates to a function on a space $X$ a function $\widehat{f}$ on a family $\Xi$ of subsets $\xi \subset X$ with the definition,

$$
\widehat{f}(\xi)=\int_{\xi} f(x) d m(x), \quad \xi \in \Xi,
$$

$d m$ being a given measure on each $\xi$. Radon's original question [9] was whether this mapping $f \rightarrow \widehat{f}$ was injective, in other words whether $f$ is determined by the integrals (1.1). Along with this injectivity problem, determining the range of the mapping $f \rightarrow \widehat{f}$ is an interesting question. A part of this question is the so-called support theorem. While the implication

$$
\operatorname{supp}(f) \text { compact } \Rightarrow \operatorname{supp}(\widehat{f}) \text { compact }
$$

(supp denoting support) will usually hold for simple reasons, the converse implication

Received April 28, 2011

MSC: 22 E30, 53 A35, 53 C35, 53 C65, 44 A12. 


$$
\operatorname{supp}(\widehat{f}) \text { compact } \Rightarrow \operatorname{supp}(f) \text { compact }
$$

is designated the support theorem (usually with extra assumption on $f$ ). Positive answers for some examples lead to various applications:

(i) An explicit description of the range $\mathcal{D}(X)^{\wedge}$ where $X$ is a Euclidean space or a symmetric space of the noncompact type $([2],[3])$. Here $\left(\mathcal{D}=\mathcal{C}_{c}^{\infty}\right)$. In the first case, $\widehat{f}$ in (1.1) is integration over hyperplanes in $X=\mathbf{R}^{n}$; in the latter case $\widehat{f}$ in (1.1) refers to integration over horocycles $\xi$ in the symmetric space $X$.

(ii) Medical application in X-ray reconstruction ([6], p.47).

(iii) Existence theorem for invariant differential equations on a symmetric space $X$ ([3], Lemma 8.1 and Theorem 8.2).

While these results rely on special methods for each case, microlocal analysis has been used e.g. by Quinto [8] for results of more general nature, requiring however stronger a priori assumptions about $f$ and its support.

For a symmetric space $X$ of the noncompact type there are two natural Radon transforms, the X-ray transform and the horocycle transform; in both cases (1.3) holds ([4], [3]). If $X$ has rank one, then a horocycle has codimension one and its interior is well defined ([1]). Thus one can raise the question of a support theorem for the X-ray transform $f \rightarrow \widehat{f}$ relative to a fixed horocycle. If $f$ is assumed exponentially decreasing, the support theorem does indeed hold ([5]). Specifically, a function on $X$ is said to be exponentially decreasing if

$$
\sup _{x} f(x) e^{m d(0, x)}<\infty
$$

for each $m>0,0 \in X$ denoting the origin and $d$ the distance.

For $X$ a hyperbolic space we consider in this note the analogous question for the horocycle transform $f \rightarrow \widehat{f}$, relative to a fixed horocycle (Theorem 2.2), extending a result by Lax and Phillips ([7]).

This paper is dedicated to the memory of Shiing-Shen Chern in appreciation of his generosity and thoughtfulness through many years. In 1959 when I was planning my 1962 book, his encouragement and advice were invaluable. He took active interest in my work developing Radon transform theory for homogeneous spaces and told me how his 1942 incidence definition for a pair of homogeneous 
spaces fits into the program. In fact, some of Radon's old results from 1917 are best understood from this point of view.

\section{The HOROCYClE TRANSFORM ON $H^{n}$}

For the support question we take the hyperbolic space $H^{n}$ with the metric

$$
d s^{2}=\frac{d x_{1}^{2}+\cdots+d x_{n}^{2}}{x_{n}^{2}}, \quad x_{n}>0 .
$$

In the metric (2.1) the geodesics are the circular arcs perpendicular to the plane $x_{n}=0$; among these are the half lines perpendicular to $x_{n}=0$. The horocycles perpendicular to these last geodesics are the planes $x_{n}=$ const. The other horocycles are the Euclidean $(n-1)$-spheres tangential to the boundary.

Let $\xi \subset \mathbf{H}^{n}$ be a horocycle in the half space model. It is a Euclidean sphere with center $\left(x^{\prime}, r\right)$ (where $\left.x^{\prime}=\left(x_{1}, \ldots x_{n-1}\right)\right)$ and radius $r$. We consider the intersection of $\xi$ with the plane through $\left(x^{\prime}, r\right)$ parallel to the $x_{n-1} x_{n}$ plane. It is the circle $\gamma: x_{n-1}=x^{\prime}+r \sin \theta, x_{n}=r(1-\cos \theta)$ where $\theta$ is the angle measured from the point of contact of $\xi$ with $x_{n}=0$. The plane $x_{n}=r(1-\cos \theta)$ intersects $\xi$ in an $(n-2)$-sphere whose points are $x^{\prime}+r \sin \theta \omega^{\prime}$ where $\omega^{\prime}=\left(\omega_{1}, \ldots, \omega_{n-1}\right)$ is a point on the unit sphere $S_{n-2}$ in $\mathbf{R}^{n-1}$. Let $d \omega^{\prime}$ be the surface element on $S_{n-2}$.

Proposition 2.1. Let $f$ be exponentially decreasing on $\mathbf{H}^{n}$. Then in the notation above,

$$
\widehat{f}(\xi)=\int_{0}^{\pi} \int_{S_{n-2}} f\left(x^{\prime}+r \sin \theta \omega^{\prime}, r(1-\cos \theta)\right) d \omega^{\prime}\left(\frac{\sin \theta}{1-\cos \theta}\right)^{n-2} \frac{d \theta}{1-\cos \theta}
$$

Proof: Since horizontal translations preserve (2.1) and commute with $f \rightarrow \widehat{f}$ we may assume $x^{\prime}=0$.

The plane $\pi_{\theta}: x_{n}=r(1-\cos \theta)$ has the non-Euclidean metric

$$
\frac{d x_{1}^{2}+\cdots+d x_{n-1}^{2}}{r^{2}(1-\cos \theta)^{2}}
$$

and the intersection $\pi_{\theta} \cap \xi$ is an $(n-2)$-sphere with induced metric

$$
\frac{r^{2} \sin ^{2} \theta\left(d \omega^{\prime}\right)^{2}}{r^{2}(1-\cos \theta)^{2}}
$$


where $\left(d \omega^{\prime}\right)^{2}$ is the metric on the $(n-2)$-dimensional unit sphere in $\mathbf{R}^{n-1}$. The non-Euclidean volume element on $\xi \cap \pi_{\theta}$ is thus

$$
\left(\frac{\sin \theta}{1-\cos \theta}\right)^{n-2} d \omega^{\prime}
$$

The non-Euclidean arc element on $\gamma$ is by $(2.1)$ equal to $d \theta /(1-\cos \theta)$. Putting these facts together (2.2) follows by integrating over $\xi$ by slices $\xi \cap \pi_{\theta}$.

Theorem 2.2. Let $\xi_{0} \subset \mathbf{H}^{n}$ be a fixed horocycle. Let $f$ be exponentially decreasing and assume

$$
\widehat{f}(\xi)=0
$$

for each horocycle $\xi$ lying outside $\xi_{0}$. Then

$$
f(x)=0 \text { for } x \text { outside } \xi_{0} .
$$

\section{Remark.}

For the case $n=3$ this is proved in Lax-Phillips [7]. As we see below, this case is an exception and the general case requires additional methods.

Proof: By homogeneity we may take $\xi_{0}$ as the plane $x_{n}=1$. Assuming $\widehat{f}(\xi)=0$ we take the Fourier transform in the $x^{\prime}$ variable of the right hand side of (2.2), in other words integrate it against $e^{-i\left\langle x^{\prime}, \eta^{\prime}\right\rangle}$ where $\eta^{\prime} \in \mathbf{R}^{n-1}$.

Then

$$
\int_{0}^{\pi} \int_{S_{n-2}} \widetilde{f}\left(\eta^{\prime}, r(1-\cos \theta)\right) e^{-i r \sin \theta\left\langle\eta^{\prime}, \omega^{\prime}\right\rangle} d \omega^{\prime}\left(\frac{\sin \theta}{1-\cos \theta}\right)^{n-2} \frac{d \theta}{1-\cos \theta}=0 .
$$

By rotational invariance the $\omega^{\prime}$ integral only depends on the norm $\left|\eta^{\prime}\right| r \sin \theta$ so we write

$$
J\left(r \sin \theta\left|\eta^{\prime}\right|\right)=\int_{S_{n-2}} e^{-i r \sin \theta\left\langle\eta^{\prime}, \omega^{\prime}\right\rangle} d \omega^{\prime}
$$

and thus

$$
\int_{0}^{\pi} \widetilde{f}\left(\eta^{\prime}, r(1-\cos \theta)\right) J\left(r \sin \theta\left|\eta^{\prime}\right|\right)\left(\frac{\sin \theta}{1-\cos \theta}\right)^{n-2} \frac{d \theta}{1-\cos \theta}=0 .
$$


Here we substitute $u=r(1-\cos \theta)$ and obtain

$$
\int_{0}^{2 r} \tilde{f}\left(\eta^{\prime}, u\right) J\left(\left(2 u r-u^{2}\right)^{1 / 2}\left|\eta^{\prime}\right|\right) \frac{r}{u^{n-1}}\left(2 u r-u^{2}\right)^{\frac{1}{2}(n-3)} d u=0 .
$$

Since the distance from the origin $(0,1)$ to $\left(x^{\prime}, u\right)$ satisfies

$$
d\left((0,1),\left(x^{\prime}, u\right)\right) \geq d((0,1),(0, u))=\int_{u}^{1} \frac{d x_{n}}{x_{n}}=-\log u
$$

so

$$
e^{d\left((0,1),\left(x^{\prime}, u\right)\right)} \geq \frac{1}{u}
$$

and since

$$
\widetilde{f}\left(\eta^{\prime}, u\right)=\int_{\mathbf{R}^{n-1}} f\left(x^{\prime}, u\right) e^{-i\left\langle x^{\prime}, \eta^{\prime}\right\rangle} d x^{\prime},
$$

we see from the exponential decrease of $f$, that the function $u \rightarrow \widetilde{f}\left(\eta^{\prime}, u\right) / u^{n-1}$ is continuous down to $u=0$.

The case $n=3$. In this simplest case (2.3) takes the form

$$
\int_{0}^{2 r} \widetilde{f}\left(\eta^{\prime}, u\right) u^{-2} J\left(\left(2 u r-u^{2}\right)^{1 / 2}\left|\eta^{\prime}\right|\right) d u=0 .
$$

We need here standard result for Volterra integral equation (cf. Yosida [10]).

Proposition 2.3. Let $a<b$ and $f \in C[a, b]$ and $K(s, t)$ of class $C^{1}$ on $[a, b] \times$ $[a, b]$. Then the integral equation

$$
\varphi(s)+\int_{a}^{s} K(s, t) \varphi(t) d t=f(s)
$$

has a unique continuous solution $\varphi(t)$. In particular, if $f \equiv 0$ then $\varphi \equiv 0$.

Corollary 2.4. Assume $K(s, s) \neq 0$ for $s \in[a, b]$. Then the equation

$$
\int_{a}^{s} K(s, t) \psi(t) d t=0 \quad \text { implies } \psi \equiv 0 .
$$


This follows from Prop. 2.3 by differentiation. Using Cor. 2.4 on (2.4) we deduce $\tilde{f}\left(\eta^{\prime}, u\right)=0$ for $u \leq 2 r$ with $2 r \leq 1$ proving Theorem 2.2 for $n=3$.

The case $n=2$. Here (2.3) leads to the generalized Abel integral equation $(0<\alpha<1)$.

$$
\int_{a}^{s} \frac{G(s, t)}{(s-t)^{\alpha}} \varphi(t) d t=f(s) .
$$

Theorem 2.5. With $f$ continuous, $G$ of class $C^{1}$ and $G(s, s) \neq 0$ for all $s \in[a, b]$, equation (2.7) has a unique continuous solution $\varphi$. In particular, $f \equiv 0 \Rightarrow \varphi \equiv 0$.

This is proved by integrating the equation against $1 /(x-s)^{1-\alpha}$ whereby the statement is reduced to Cor. 2.4 (cf. Yosida, loc.cit.).

This proves Theorem 2.2 for $n=2$.

The general case. Here the parity of $n$ makes a difference. For $n$ odd we just use the following lemma.

Lemma 2.6. Assume $\varphi=C^{1}([a, b])$ and that $K(s, t)$ has all derivatives with respect to $s$ up to order $m-2$ equal to 0 on the diagonal $(s, s)$. Assume the $(m-1)^{\text {th }}$ order derivative is nowhere 0 on the diagonal. Then (2.6) still holds.

In fact, by repeated differentiation of (2.6) one can show that (2.5) holds with a kernel

and $f \equiv 0$.

$$
\frac{K^{(m)}(s, t)}{\left\{K_{s}^{(m-1)}(s, t)\right\}_{t=s}}
$$

This lemma proves Theorem 2.2 for $n$ odd. For $n$ even we write (2.3) in the general form

$$
\int_{0}^{s} F(u) H\left(\left(s u-u^{2}\right)^{1 / 2}\right)\left(s u-u^{2}\right)^{\frac{1}{2}(n-3)} d u=0 \quad n \quad \text { even } \geq 2,
$$

where $H(0) \neq 0$.

Theorem 2.7. Assume $F \in C([0,1])$ satisfies (2.8) for $0 \leq s \leq 1$ and $H \in \mathrm{e}^{\infty}$ arbitrary with $H(0) \neq 0$. Then $F \equiv 0$ on $[0,1]$. 
Proof: We proceed by induction on $n$, the case $n=2$ being covered by Theorem 2.5. We assume the theorem holds for $n$ and any function $H$ satisfying $H(0) \neq 0$. We consider (2.8) with $n$ replaced by $n+2$ and take $d / d s$. The result is with $H_{1}(x)=H^{\prime}(x) x+(n-1) H(x)$,

$$
\int_{0}^{s} F(u) u H_{1}\left(\left(s u-u^{2}\right)^{\frac{1}{2}}\right)\left(s u-u^{2}\right)^{\frac{1}{2}(n-3)} d u=0 .
$$

Since $H_{1}(0) \neq 0$ we conclude $F \equiv 0$ by induction. This finishes the proof of Theorem 2.2.

\section{REFERENCES}

[1] Faraut, J., Un théorème de Paley-Wiener pour la transformation de Fourier sur un espace Riemannian symétrique de rang un, J. Funct. Anal. 49 (1982), 230-268.

[2] Helgason, S., The Radon transform on Euclidean spaces, compact two-point homogeneous spaces and Grassmann manifolds, Acta Math. 113 (1965), 153-180.

[3] The surjectivity of invariant differential operators on symmetric spaces, Ann. of Math. 98 (1973), 451-480.

[4] "The X-ray transform on a symmetric space," in: Proc. Conf. on Differential Geometry and Global Analysis, Berlin, 1979, Lecture Notes in Math. No. 838, SpringerVerlag, New York, 1980.

[5] Some results on Radon transforms, Huygens' principle and X-ray transforms, Contemp. Math. Vol. 63 (1987).

[6] The Radon Transform, $2^{\text {nd }}$ edition, Birkhäuser, Basel and Boston, 1999.

[7] Lax, P. and Phillips, R.S., Translation representations for the solution of the nonEuclidean wave equation, Comm. Pure Appl. Math. 32 (1979), 617-667.

[8] Quinto, E.T., Helgason's support theorem and spherical Radon transforms, Contemp. Math., 2008.

[9] Radon, J., Über die Bestimmung von Funktionen durch ihre Integralwerte längs gewisser Mannigfaltigkeiten, Ber. Verh. Sächs. Akad. Wiss. Leipzig. Math. Nat. Kl. 69 (1917), 262-277.

[10] Yosida, K. Lectures on Differential and Integral Equations, Interscience, New York, 1960.

Sigurdur Helgason

Department of Mathematics

Massachusetts Institute of Technology

Cambridge, MA 02139

E-mail: helgason@mit.edu 